\title{
Neuroendocrine and epithelial phenotypes in small-cell lung cancer: implications for metastasis and survival in patients
}

\author{
R Stovold ${ }^{1,2,5}, \mathrm{~S}$ L Meredith ${ }^{1,5}, \mathrm{~J} \mathrm{~L} \mathrm{Bryant}{ }^{1}, \mathrm{M} \mathrm{Babur}^{3}, \mathrm{~K} \mathrm{~J} \mathrm{Williams}{ }^{3}, \mathrm{E} \mathrm{J} \mathrm{Dean}{ }^{2}, \mathrm{C}$ Dive $^{2}, \mathrm{~F} \mathrm{H}$ Blackhall $^{4}$ and \\ A White ${ }^{*, 1,3}$ \\ ${ }^{1}$ Faculty of Life Sciences, Manchester Academic Health Sciences Centre, University of Manchester, 3.016 AV Hill Building, \\ Manchester M13 9PT, UK; ${ }^{2}$ Paterson Institute for Cancer Research, University of Manchester, Manchester M20 4BX, UK; ${ }^{3}$ Faculty of \\ Medical and Human Sciences, Manchester Academic Health Sciences Centre, University of Manchester, Stopford Building, \\ Manchester M13 9PT, UK and ${ }^{4}$ The Christie NHS Foundation Trust, Manchester M20 4BX, UK
}

Background: Small-cell lung cancer (SCLC) has a very aggressive clinical course with early metastasis. This study investigated how the distinctive neuroendocrine characteristics contribute to disease progression and invasion in human SCLC.

Methods: The neuroendocrine phenotype (pro-opiomelanocortin (POMC)) was quantified by ELISA in blood samples from 43 SCLC patients. The neuroendocrine (POMC, chromogranin A, neuron-specific enolase, NCAM) and epithelial (cytokeratin and E-cadherin) phenotypes were investigated, using ELISA and immunocytochemistry/immunohistochemistry.

Results: In SCLC patients, $16 \%$ had elevated circulating POMC, which was associated with significantly worse survival $(P=0.02)$ and liver metastases $(P=0.004)$. In addition, POMC correlated with epithelial-positive circulating tumour cells $(P=0.0002)$. In a panel of SCLC cell lines, all POMC-secreting cell lines expressed cytokeratin (40\% of total). Even after cloning, DMS 79 cells expressed both neuroendocrine and epithelial markers. DMS 79 xenografts secreted POMC into the blood, which mirrored the tumour volume. These xenografts expressed both neuroendocrine and epithelial phenotypes in all tumours, with both phenotypes prevalent in cells invading the surrounding tissue.

Conclusion: Both neuroendocrine and epithelial phenotypes coexist in human SCLC tumours in vitro and in vivo and this persists in invading tumour cells. In patients, POMC secretion predicts poor survival and liver metastases, suggesting a crucial role of the neuroendocrine phenotype.

In industrialised countries, lung cancer is the leading cause of cancer death (Jemal et al, 2009). Small-cell lung cancer (SCLC) accounts for approximately $10-15 \%$ of all lung cancer cases and is characterised by an aggressive clinical course with invariable resistance to chemotherapy despite initially high response rates (Riaz et al, 2012). There has been little improvement in survival over the past few decades, suggesting a greater understanding of the molecular mechanisms underpinning the dismal prognosis of SCLC is urgently required.
Recent studies in mice suggest cooperativity between SCLC cells with epithelial and neuroendocrine characteristics, and that the latter are the critical cell type for metastatic formation in SCLC, at least in this mouse model (Calbo et al, 2011). Our studies of circulating human tumour cells also suggest distinct cellular heterogeneity within SCLC (Hou et al, 2011). From these studies, it is becoming clear that SCLC exhibits a heterogeneous phenotype, of which further investigations are warranted.

\footnotetext{
*Correspondence: Professor A White; E-mail: anne.white@manchester.ac.uk

${ }^{5}$ These authors contributed equally to this work.
}

Received 4 December 2012; revised 12 February 2013; accepted 20 February 2013; published online 21 March 2013 
Pathological confirmation of SCLC has routinely utilised various neuroendocrine peptides, such as CD56 (NCAM), chromogranin A (CgA) and synaptophysin (Travis et al, 2004). In healthy individuals, neuroendocrine cells are prominent in foetal lungs and gradually decrease in number as the lungs mature. Hence, it is suggested that neuroendocrine cells may have a role in lung development and repair and have been shown to produce numerous hormones (Travis, 1999). In patients with SCLC, it is thought that some of these 'hormones' may act in the metastatic process by autocrine activity or by cell-cell interactions. Several of these neuroendocrine hormones/peptides can be measured in SCLC patients and have been utilised as biomarkers of the disease and the target of therapeutic trials (e.g. BB-10901 studies targeting CD56 (British Biotech Pharmaceuticals trial BBIO-C10/IVB/001)) (Abidin et al, 2010).

Pro-opiomelanocortin (POMC) is a prohormone expressed primarily in the pituitary, hypothalamus and skin, and is also found in neuroendocrine cells of the lung. In the pituitary, POMC undergoes intracellular processing by prohormone convertases (PC) to produce a number of hormones, most importantly adrenocorticotrophin (ACTH), (Stovold et al, 2012).

Early studies in patients with SCLC suggested that ACTH was one of the most prevalent neuropeptides secreted by SCLC tumours (Ratcliffe et al, 1982; Clark and White, 1994). This is supported by the observation of Cushingoid symptoms in a proportion of patients, who are therefore deemed to have the ectopic ACTH syndrome. These studies use less specific assays and imply ACTH is secreted in high levels by SCLC tumours. However, later studies have shown that it is, in fact, POMC that is found in the circulation of patients with SCLC, and that it is a putative marker of the disease (Oliver et al, 2003). There is also evidence from these early studies that in patients without clinical symptoms, SCLC tumours secrete these ACTH-related peptides (Trump et al, 1982).

The aims of this study were to characterise patients with SCLC for expression of the novel neuroendocrine marker, POMC, and to use POMC to investigate whether there are distinct neuroendocrine or epithelial characteristics in human SCLC tumours and in particular whether this defines the tumour cells responsible for invasion.

\section{MATERIALS AND METHODS}

Blood collection from SCLC patients. Blood samples were collected, at The Christie NHS Foundation Trust, from patients with limited- and extensive-stage SCLC, who had given written informed consent for the study according to an ethically approved protocol. Blood samples were collected using standard phlebotomy techniques with the vacutainer system (Becton Dickinson, Franklin Lanes, NJ, USA). Plasma samples were collected in tubes containing lithium-heparin for the POMC ELISA described below. Enumeration of circulating tumour cells (CTCs) was conducted using the CellSearch platform (Veridex, Raritan, NJ, USA) as described previously (Tibbe et al, 1999). All patients were Caucasian smokers. Metastases were determined at diagnosis, that is, before treatment in the liver, bone, lungs, adrenals, lymph nodes, brain, thyroid, pleural cavity, spleen and pancreas.

SCLC cell lines. All COR cell lines were kindly donated by Dr P Twentyman (MRC Clinical Oncology and Radiotherapeutics Unit, Cambridge, UK) and established and characterised by BaillieJohnson et al (1985). DMS cell lines were supplied by Dr Pettengill (Dartmouth Medical School, Hanover, NH, USA). NCI cell lines were obtained from ATCC (Manassas, VA, USA). HC12 cells were donated by Dr G Duchesne (Ludwig Institute of Cancer Research, Sutton, Surrey, UK). Small-cell lung cancer cell lines grow as suspended aggregates (excluding DMS 53 cells that grow as an adherent monolayer) and were cultured in RTISS media (RPMI$1640+\mathrm{L}$-glutamine supplemented with $2.5 \% \mathrm{FBS}, 5 \mu \mathrm{g} \mathrm{ml}^{-1}$ insulin, $10 \mu \mathrm{g} \mathrm{ml}^{-1}$ transferin, $30 \mathrm{nM}$ sodium selenite and 1\% HEPES buffer) (White et al, 1989). Cells were seeded at $5 \times 10^{4}$ per $\mathrm{ml}$ and cultured for 10 days before harvesting media and cell pellets for analysis.

Xenograft studies. All procedures involving animals were performed in accordance with the UK Home Office Animal (Scientific Procedures) Act, 1986, and approved by the local University of Manchester Ethical Review Committee. DMS 79 cells were injected subcutaneously into nude mice at $5 \times 10^{6}$ cells per mouse in $0.1 \mathrm{ml}$ of serum-free RPMI-1640 medium with the addition of $50 \%$ Matrigel. Tumours were allowed to reach a volume of $1000 \mathrm{~mm}^{3}$ before they were excised and half-snap frozen and half-formalin fixed and -paraffin wax embedded. Blood samples (maximum $80 \mu \mathrm{l}$ ) were taken by tail nick at day 0 and then every 7 days from day 13. NCI-H526 xenografts were prepared in the same way, excluding tail nick sampling. However, blood samples were taken at death when tumours reached $1000 \mathrm{~mm}^{3}$ by cardiac puncture. Mouse plasma was analysed for POMC by ELISA (see below).

Immunocytochemistry/immunohistochemistry. DMS 79 and NCI-H526 cells were prepared as cytospins on polysine-coated slides. A549 NSCLC cells were grown on cover slips and used as a negative control, as this cell line does not express neuroendocrine markers. Cells were subsequently fixed and stained using the Dako chromagen envision system (Cambridge, UK). The primary antibodies to CD56 (N-CAM), CK (AE1/AE3; a mixture of two monoclonal antibodies recognising CK 10,13-16 and 19 and CK $1-8$, respectively), chromogranin $\mathrm{A}$, neuron-specific enolase and E-cadherin were mouse monoclonals, which were obtained from Dako. The primary monoclonal antibody recognising POMC (N1C11) was produced in our labs and purified using protein A (Crosby et al, 1988). Slides were examined by light microscopy.

Slices of $5 \mu \mathrm{m}$ from formalin-fixed wax-embedded DMS 79 tumours were stained for POMC (N1C11), NSE, CK (AE1/AE3) and vimentin (mouse monoclonal; Dako) using the Dako chromagen envision system. Antigen retrieval was performed on all slides using citrate buffer $(\mathrm{pH} 6)$ at $95^{\circ} \mathrm{C}$ for $30 \mathrm{~min}$. Staining was analysed by light microscopy.

POMC ELISA. Pro-opiomelanocortin levels were measured using a specific two-site ELISA. Pro-opiomelanocortin was measured in 9 patients using a research assay, but following validation, the remaining samples from 34 patients were measured in a GCP validated assay. Both assays followed the same protocol, which was based on the immunoradiometric assay, described previously (Crosby et al, 1988), using either $40 \mu \mathrm{l}$ (validated assay) or $100 \mu \mathrm{l}$ (research assay) of plasma run in duplicate. The ELISA utilises purified human POMC as a standard (prepared as described below). A monoclonal antibody (A1A12), which recognises the ACTH 10-18 region of POMC, is coated onto an ELISA plate, and a monoclonal antibody (N1C11), which recognises the $\gamma$-MSH region of POMC, is biotin labelled and used as the detection antibody. Binding of both antibodies is required to generate a signal in the assay. Therefore, the POMC assay does not crossreact with ACTH, but does crossreact 100\% with POMC and proACTH. The lower limit of assay sensitivity during the study was $15 \mathrm{pmoll}^{-1}$ for the research assay and $30 \mathrm{pmoll}^{-1}$ for the GCP validated assay.

POMC purification. We previously produced a monoclonal antibody (N1C11) to the $\gamma$-MSH region of POMC (Crosby et al, 1988). This antibody was coupled to a solid-phase support (Sephacryl S-300; GE Healthcare, Yorkshire, UK) as described previously (Crosby et al, 1988) or affinity purification of POMC. The solid-phase antibody was stored at $4{ }^{\circ} \mathrm{C}$. Supernatant media 
from confluent DMS 79 cells were collected by centrifugation and used immediately or frozen at $-20^{\circ} \mathrm{C}$. Aliquots of media $(400 \mathrm{ml})$ were filtered through a $0.45-\mu \mathrm{m}$ membrane and incubated overnight at $4{ }^{\circ} \mathrm{C}$ with $10 \mathrm{ml} 50 \% \mathrm{~N} 1 \mathrm{C} 11$ solid phase on a shaking platform. N1C11 solid phase was collected by centrifugation and washed three times with PBS containing polypep (Sigma, Dorset, $\mathrm{UK})$. Pro-opiomelanocortin peptide was eluted with citrate $(\mathrm{pH}$ 3.0) and neutralised with $1 \mathrm{M}$ Tris. Purified POMC was analysed for POMC as described above, and then stored at $-80^{\circ} \mathrm{C}$.

CK ELISA. Full-length and caspase-cleaved CK18 fragments were measured in the media from SCLC cell lines using the M65 ELISA kit (Peviva, Bromma, Sweden) according to the manufacturer's instructions.

CK gene expression. RNA was extracted from SCLC cells to measure the CK18 gene expression by reverse transcription-PCR using the RNEasy kit according to the manufacturer's instructions (Qiagen, West Sussex, UK). cDNA synthesis was carried out using the QuantiTect Reverse Transcription Kit (Qiagen). Primers were obtained from Eurofins MWG (London, UK) and were designed across exon boundaries. Polymerase chain reaction products were resolved on a $2 \%$ agarose gel containing $0.5 \mu \mathrm{g} \mathrm{ml}^{-1}$ ethidium bromide and run in Tris-acetate EDTA buffer at $90 \mathrm{~V}$ for $50 \mathrm{~min}$. Gels were then visualised under UV light $(254 \mathrm{~nm})$.

Data analysis. All image capture and analysis was conducted using an Axioskop upright brightfield microscope and Axiovision software (Zeiss Microscopy and Imaging, Cambridge, UK). All statistical analyses were performed using the GraphPad Prism software (version 5; GraphPad, La Jolla, CA, USA).

\section{RESULTS}

Circulating POMC concentrations in patients with SCLC. Proopiomelanocortin was measured in the circulation of 43 patients with SCLC as a marker for the neuroendocrine phenotype. Levels were in excess of the upper limit of the normal range $\left(>100 \mathrm{pmoll}^{-1}\right)$ in $16 \%$ of patients (mean POMC $136 \mathrm{pmoll}^{-1}$, range $15-1500 \mathrm{pmoll}^{-1}$ ) (Table 1$)$.

There was no significant difference in levels of circulating POMC in the extensive-stage disease group (median $37 \mathrm{pmoll}^{-1}, n=27$ ) compared with the limited-stage disease group $\left(30 \mathrm{pmoll}^{-1}\right.$, $n=16$ ) (Figure 1A). There was no difference in circulating POMC levels between patients with and without the presence of metastases (metastases median, $33.5 \mathrm{pmol}^{-1}, n=28$; no metastases median, $30 \mathrm{pmoll}^{-1}, n=15$ ) (Figure 1B). However, POMC levels were significantly higher in patients with liver metastases compared with those patients with metastases at other sites (see Table 1 for other sites analysed for metastases) (liver metastases median, $54 \mathrm{pmoll}^{-1}$, $n=17$; other sites of metastases median, $30 \mathrm{pmoll}^{-1}, n=11$; $P<0.01$ one-way ANOVA) Figure 1C.

Circulating POMC has a significant correlation with CTCs and with effect on overall survival. Circulating tumour cells were detected using the CellSearch system (Veridex), which identifies cells positive for EpCam (epithelial cell adhesion molecule) and CK. Therefore, all CTCs are considered epithelial in phenotype. This is in support of patient data showing that almost all SCLC biopsies contain CK (Travis et al, 2004). There was a significant correlation between CTC number (and therefore epithelial phenotype) and POMC in SCLC patients (Spearman's correlation $P=0.0002, n=43$ ) (Figure 2A). Pro-opiomelanocortin significantly correlated with the adverse prognostic factor lactate dehydrogenase (LDH) $(P=0.003, n=43$ data not shown). Smallcell lung cancer patients with elevated circulating POMC $\left(>100\right.$ pmoll $\left.^{-1}, n=7\right)$ had a significantly worse overall survival by univariate analysis compared to those with normal POMC $\left(<100\right.$ pmoll $^{-1}, n=36, P=0.02$ ) (Figure 2B).
Neuroendocrine and epithelial characteristics of SCLC cell lines. In addition to assessing the epithelial and neuroendocrine phenotypes of SCLC in patients, a panel of SCLC cell lines were also investigated. This was achieved by measuring the release of POMC as the neuroendocrine marker and by analysing CK release from the cells as the epithelial marker (Figure 3). Of these cell lines, $40 \%$ (6 out of 15) released POMC and 80\% (12 out of 15) CK. All of the POMC-secreting cells also released CK. Of the remaining cell lines, most released CK and only three had neither marker. Both POMC and CK were also measured in cell extracts (data not shown). Cell lines not secreting these markers into the culture medium also had no detectible intracellular peptide levels. Cytokeratin gene expression was also investigated in the SCLC cell lines (Figure 3). Results showed that all cell lines expressed CK18 mRNA, suggesting that levels in the media of 3 out of 15 cell lines were below the limit of detection by ELISA and not that these cell lines lack epithelial characteristics.

A clonal cell line was derived from the SCLC cell line, DMS 79, and investigated for neuroendocrine and epithelial markers by immunocytochemistry. The cell line stained positive for POMC and for CK. It is of note that there was heterogeneity even within this cloned cell line (Figure 4). The SCLC cell line was also positive for other neuroendocrine markers (CgA, NSE and CD56 (NCAM)) and again there was heterogeneity in expression.

In addition to the DMS 79 cell line, a non-POMC-secreting SCLC cell line, NCI-H526, was investigated for neuroendocrine and epithelial markers and was found to be positive for CK and E-cadherin. It was also positive for CgA, NSE, CD56 (NCAM), but not POMC (Figure 4). This further supports previous work identifying a dual neuroendocrine and epithelial phenotype of SCLC. It also demonstrates the presence of heterogeneity of the neuroendorine phenotype between SCLC cell lines.

Neuroendocrine and epithelial characteristics of the SCLC xenograft model. The DMS 79 SCLC cell line exhibited a heterogeneous phenotype in vitro with positive staining for neuroendocrine and epithelial markers. Therefore, this cell line was selected for injection subcutaneously into nude mice to analyse its phenotype when grown as an in vivo xenograft. Our results show that POMC is detectable when the tumour volume is small (at $200 \mathrm{~mm}^{3}$ ) and is directly related to tumour burden (Figure 5A). At the time of tumour harvest, circulating POMC had increased 11-fold compared with a non-tumour control (Figure 5B). When compared with a non-POMC-secreting SCLC tumour control, POMC levels were also significantly higher (no xenograft, $145 \mathrm{pmoll}^{-1} ; \quad \mathrm{NCI}-\mathrm{H} 526,240 \mathrm{pmoll}^{-1} ;$ and DMS 79, $1580 \mathrm{pmoll}^{-1} ; P<0.001$ ) (Figure 5B).

In the DMS 79 xenografts, there were central pools of necrosis present within the tumours, which highlighted the aggressive nature of the tumour (Figure 6). Consistent with the findings of the in vitro studies, the xenografts had positive staining for POMC, NSE and CK (Figures 6A-C), but were negative for the mesenchymal marker vimentin (Figure 6D). In other tumour types, epithelial-to-mesenchymal transition is associated with metastasis, but it is not clear whether this occurs in SCLC and how the neuroendocrine-positive cells are involved in human SCLC metastasis. Pro-opiomelanocortin-positive tumour cells were seen to be invading into the tumour fibrotic capsule and muscle layers outside the tumour, which are visible on the edge of the xenografts (Figure 6E). These invading tumour cells were also positive for CK and NSE.

\section{DISCUSSION}

In this study, we used POMC as a novel neuroendocrine prohormone marker, which can be analysed in the patient blood. 
Table 1. Characteristics of SCLC patients and circulating POMC levels $\left(\right.$ pmol $\mathrm{I}^{-1}$ )

\begin{tabular}{|c|c|c|c|c|c|c|}
\hline Patient & Gender & $\begin{array}{c}\text { Age } \\
\text { (years) }\end{array}$ & PS & $\begin{array}{l}\text { Disease } \\
\text { stage }\end{array}$ & $\begin{array}{l}\text { Treatment } \\
\text { regimen }\end{array}$ & $\begin{array}{c}\text { Circulating } \\
\text { POMC } \\
\left(\text { pmoll }^{-1}\right)\end{array}$ \\
\hline
\end{tabular}

Metastases

\begin{tabular}{|c|c|c|c|c|c|c|c|c|c|c|c|c|}
\hline & & & & & & & Liver & Bone & Lung & Adrenal & $\begin{array}{l}\text { Lymph } \\
\text { nodes }\end{array}$ & $\begin{array}{l}\text { Pleural } \\
\text { effusion }\end{array}$ \\
\hline 1 & M & 64 & 2 & $\mathrm{~L}$ & Cis/etop & 30 & 0 & 0 & 0 & 0 & 0 & 0 \\
\hline 2 & M & 79 & 2 & $\mathrm{E}$ & Carb/etop & 443 & 1 & 0 & 0 & 0 & 0 & 0 \\
\hline 3 & M & 79 & 1 & $\mathrm{E}$ & Carb/etop & 128 & 1 & 1 & 0 & 0 & 0 & 0 \\
\hline 4 & M & 55 & 2 & $\mathrm{E}$ & Carb/etop & 30 & 0 & 0 & 1 & 0 & 0 & 0 \\
\hline 5 & $\mathrm{~F}$ & 69 & 2 & $\mathrm{E}$ & Carb/etop & 104 & 1 & 0 & 0 & 0 & 1 & 0 \\
\hline 6 & $\mathrm{~F}$ & 69 & 1 & L & Carb/etop & 30 & 0 & 0 & 0 & 0 & 0 & 0 \\
\hline 7 & M & 52 & 3 & $\mathrm{E}$ & Carb/etop & 60 & 1 & 1 & 0 & 0 & 0 & 0 \\
\hline 8 & $\mathrm{~F}$ & 67 & 1 & $\mathrm{E}$ & Carb/etop & 30 & 0 & 0 & 0 & 0 & 1 & 0 \\
\hline 9 & M & 69 & 1 & $\mathrm{E}$ & Carb/etop & 30 & 0 & 1 & 0 & 0 & 1 & 1 \\
\hline 10 & M & 69 & 3 & $E$ & Carb/etop & 30 & 1 & 0 & 0 & 0 & 0 & 1 \\
\hline 11 & $\mathrm{~F}$ & 62 & 2 & $\mathrm{E}$ & Carb/etop & 30 & 0 & 0 & 1 & 1 & 0 & 0 \\
\hline 12 & $F$ & 76 & 0 & L & Carb/etop & 30 & 0 & 0 & 0 & 0 & 0 & 0 \\
\hline 13 & $\mathrm{~F}$ & 82 & 0 & $\mathrm{~L}$ & Carb/etop & 30 & 0 & 0 & 0 & 0 & 0 & 0 \\
\hline 14 & M & 68 & 0 & $E$ & Carb/etop & 30 & 0 & 0 & 1 & 0 & 0 & 0 \\
\hline 15 & $\mathrm{~F}$ & 55 & 1 & $E$ & Carb/etop & 37 & 1 & 0 & 0 & 0 & 0 & 0 \\
\hline 16 & M & 62 & 1 & $\mathrm{E}$ & Carb/etop & 30 & 0 & 0 & 0 & 0 & 1 & 0 \\
\hline 17 & $\mathrm{~F}$ & 57 & 1 & $E$ & Carb/etop & 30 & 1 & 0 & 1 & 0 & 1 & 1 \\
\hline 18 & M & 68 & 1 & $\mathrm{E}$ & Carb/etop & 52 & 1 & 0 & 0 & 0 & 0 & 0 \\
\hline 19 & $\mathrm{~F}$ & 63 & 1 & $E$ & Carb/etop & 52 & 0 & 0 & 1 & 0 & 1 & 0 \\
\hline 20 & $\mathrm{~F}$ & 64 & 2 & $E$ & Carb/etop & 30 & 1 & 0 & 1 & 1 & 0 & 0 \\
\hline 21 & M & 69 & 1 & $\mathrm{E}$ & Carb/etop & 67 & 1 & 0 & 0 & 0 & 0 & 0 \\
\hline 22 & M & 67 & 0 & $\mathrm{~L}$ & Cis/etop & 30 & 0 & 0 & 0 & 0 & 0 & 0 \\
\hline 23 & $\mathrm{~F}$ & 53 & 1 & L & Carb/etop & 30 & 0 & 0 & 0 & 0 & 0 & 0 \\
\hline 24 & $\mathrm{~F}$ & 61 & 1 & $E$ & Carb/etop & 30 & 1 & 0 & 0 & 0 & 1 & 0 \\
\hline 25 & $\mathrm{~F}$ & 65 & 1 & L & Carb/etop & 30 & 0 & 0 & 0 & 0 & 0 & 0 \\
\hline 26 & M & 72 & 0 & $E$ & Carb/etop & 253 & 1 & 0 & 0 & 0 & 0 & 0 \\
\hline 27 & $\mathrm{~F}$ & 71 & 1 & L & Cis/etop & 30 & 0 & 0 & 0 & 0 & 0 & 0 \\
\hline 28 & $\mathrm{~F}$ & 68 & 0 & $E$ & Carb/etop & 30 & 0 & 0 & 1 & 0 & 1 & 0 \\
\hline 29 & $\mathrm{~F}$ & 81 & 3 & $\mathrm{E}$ & Carb & 915 & 1 & 0 & 0 & 0 & 0 & 0 \\
\hline 30 & $\mathrm{~F}$ & 76 & 1 & L & Carb/etop & 48 & 0 & 0 & 0 & 0 & 0 & 0 \\
\hline 31 & $\mathrm{~F}$ & 64 & 3 & $E$ & Carb/etop & 30 & 1 & 0 & 0 & 1 & 0 & 0 \\
\hline 32 & $\mathrm{~F}$ & 66 & 3 & $\mathrm{~L}$ & Carb/etop & 30 & 0 & 0 & 0 & 0 & 1 & 0 \\
\hline 33 & $\mathrm{~F}$ & 65 & 2 & $\mathrm{~L}$ & Carb/etop & 59 & 0 & 0 & 0 & 0 & 0 & 0 \\
\hline 34 & $\mathrm{~F}$ & 68 & 2 & $\mathrm{E}$ & Carb/etop & 37 & 0 & 0 & 0 & 0 & 0 & 1 \\
\hline 35 & M & 76 & 1 & $\mathrm{~L}$ & Carb & 37 & 0 & 0 & 0 & 0 & 0 & 0 \\
\hline 36 & $\mathrm{~F}$ & 81 & 2 & L & Carb/etop & 47 & 0 & 0 & 0 & 0 & 0 & 0 \\
\hline 37 & $\mathrm{~F}$ & 66 & 1 & L & Cis/etop & 36 & 0 & 0 & 0 & 0 & 0 & 0 \\
\hline 38 & $\mathrm{~F}$ & 70 & 1 & $\mathrm{~L}$ & Cis/etop & 38 & 0 & 0 & 0 & 0 & 0 & 0 \\
\hline 39 & M & 56 & 2 & $\mathrm{E}$ & VAC & 54 & 1 & 1 & 1 & 0 & 1 & 0 \\
\hline 40 & $\mathrm{~F}$ & 82 & 1 & $\mathrm{~L}$ & Carb/etop & 1216 & 0 & 0 & 0 & 0 & 0 & 0 \\
\hline 41 & $M$ & 84 & 2 & E & no chemo & 52 & 1 & 0 & 0 & 1 & 1 & 0 \\
\hline 42 & $\mathrm{~F}$ & 53 & 1 & $\mathrm{E}$ & Carb/etop & 15 & 0 & 1 & 0 & 1 & 0 & 0 \\
\hline 43 & $M$ & 56 & 3 & $E$ & Carb & 1500 & 1 & 0 & 0 & 0 & 0 & 0 \\
\hline
\end{tabular}

Abbreviations: $\mathrm{Carb}=$ carboplatin; $\mathrm{Cis}=$ cisplatin; $\mathrm{e}=$ extensive disease; $\mathrm{Etop}=$ etoposide; $\mathrm{f}=$ female; $\mathrm{I}=$ limited disease; $\mathrm{m}=\mathrm{male} ; \mathrm{POMC}=$ pro-opiomelanocortin; $\mathrm{PS}=$ performance status; $\mathrm{VAC}=$ vincristine, adriamycin, cyclophosphamide. Metastases: $0=$ no metastases; $1=$ metastases determined at diagnosis. Other areas of metastases examined were: spleen -1 patient with metastasis; pancreas -1 patient with metastasis. No metastases were found in the brain or thyroid of any of the patients. All patients were Caucasian and smokers. 

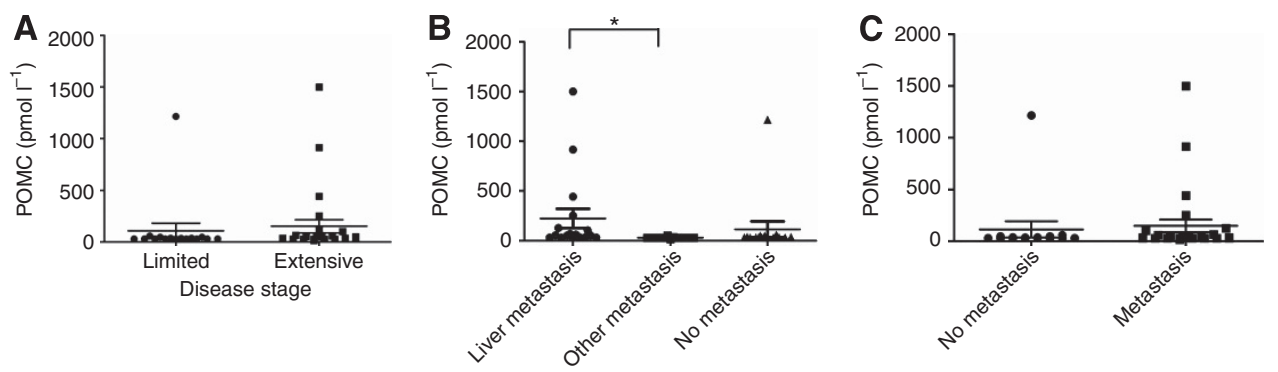

Figure 1. Comparison of POMC with stage and metastasis. (A) POMC according to disease stage; (B) POMC according to metastasis; (C) POMC according to site of metastasis (liver metastasis vs other metastasis, $P=0.004$ ).

A

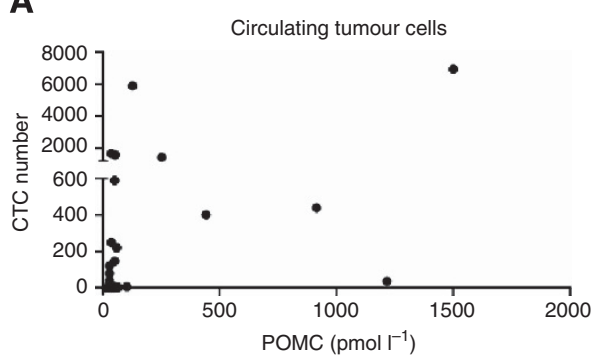

B

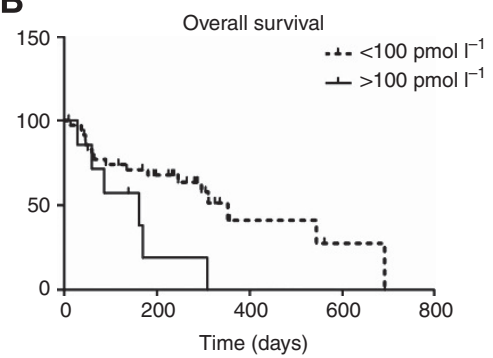

Figure 2. (A) Correlation between circulating tumour cell (CTC) number and circulating POMC in SCLC patients (Spearman correlation, $P=0.0002, n=43)$. (B) Overall survival according to circulating POMC levels. Broken line $<100$ pmol $I^{-1}$, solid line $>100$ pmoll $I^{-1}$. SCLC patients with elevated circulating POMC ( $\left.>100 \mathrm{pmoll}^{-1}\right)$ had a significantly worse overall survival compared to those with normal POMC $\left(<100\right.$ pmol I ${ }^{-1}$, $P=0.02$, Mantel-Cox test: $<100$ pmoll $^{-1}, n=36,>100$ pmoll $\left.^{-1}, n=7\right)$.

\begin{tabular}{|c|c|c|c|c|c|c|c|c|}
\hline Cell line & DMS79 & COR-L24 & COR-L103 & COR-L88 & COR L31 & DMS153 & DMS53 & COR-L27 \\
\hline $\begin{array}{c}\text { POMC } \\
(\mathrm{pmol} \mathrm{I-1})\end{array}$ & 3274 & 1753 & 198 & 34 & 33 & 24 & 0 & 0 \\
\hline${\mathrm{CK}\left(\mathrm{U} \mathrm{I}^{-1}\right)}^{-4}$ & 43 & 19 & 187 & 361 & 328 & 179 & 293 & 186 \\
\hline
\end{tabular}

\begin{tabular}{|c|c|c|c|c|c|c|c|}
\hline Cell line & COR-L32 & COR-L42 & COR-L47 & COR-L51 & COR-L99 & NCI-H82 & HC12 \\
\hline $\begin{array}{c}\text { POMC } \\
(\mathrm{pmol} \mathrm{I-1)}\end{array}$ & 0 & 0 & 0 & 0 & 0 & 0 & 0 \\
\hline${\mathrm{CK}\left(\mathrm{Ul}^{-1}\right)}^{-1}$ & 61 & 0 & 34 & 0 & 61 & 0 & 82 \\
\hline
\end{tabular}

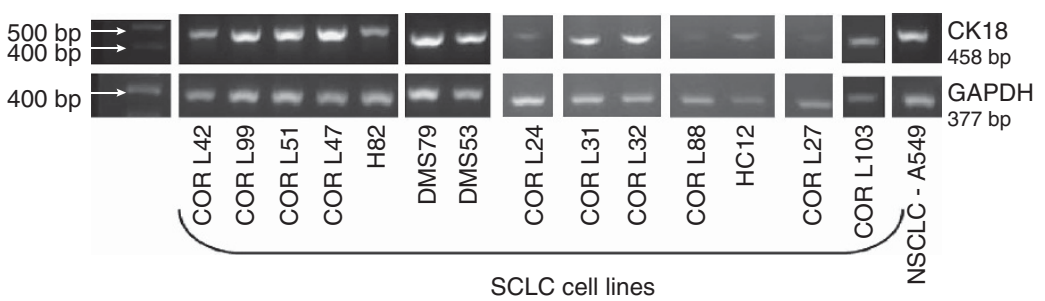

Figure 3. POMC and cytokeratin (CK) release in a panel of 16 SCLC cell lines. All SCLC cell lines were seeded at $5 \times 10^{4} \mathrm{ml}^{-1}$. Supernatants were taken at day 10 and POMC quantified by ELISA (developed in our lab) and CK by M65 ELISA (Peviva). Samples under the detection limit of the assay (15 pmol// POMC and $11 \mathrm{UI}^{-1} \mathrm{CK}$ ) are displayed as 0 . Results represent three independent cultures for each cell line and were assayed in duplicate and normalised to $10^{6}$ total cells. $\mathrm{NCl}-\mathrm{H} 526$ cells have previously been shown to release CK, but not POMC. CK18 gene expression in SCLC cell lines. RNA was extracted from cell pellets grown in RTISS media for 10 days. NSCLC cells A549 were used as a positive control. GAPDH was used as a control for consistent mRNA levels between cell lines. CK18 band is located at 458 bp. All results are representative of three independent cultures of SCLC cell lines.

We found that in patients with SCLC, only a subset have POMC in their circulation, but this correlated with CK- and E-cadherinpositive (i.e. epithelial) CTCs. Elevated circulating POMC also correlated with liver metastases, $\mathrm{LDH}$ and poor survival. This indicates that POMC may be a neuroendocrine marker of invasion and metastasis in this subset of SCLC patients. However, in a panel of SCLC cell lines those that were positive for POMC also had an epithelial phenotype, and even in a clonal cell line, both markers were expressed. The dual phenotype also persisted in an in vivo xenograft model of human SCLC. We found consistent staining for 

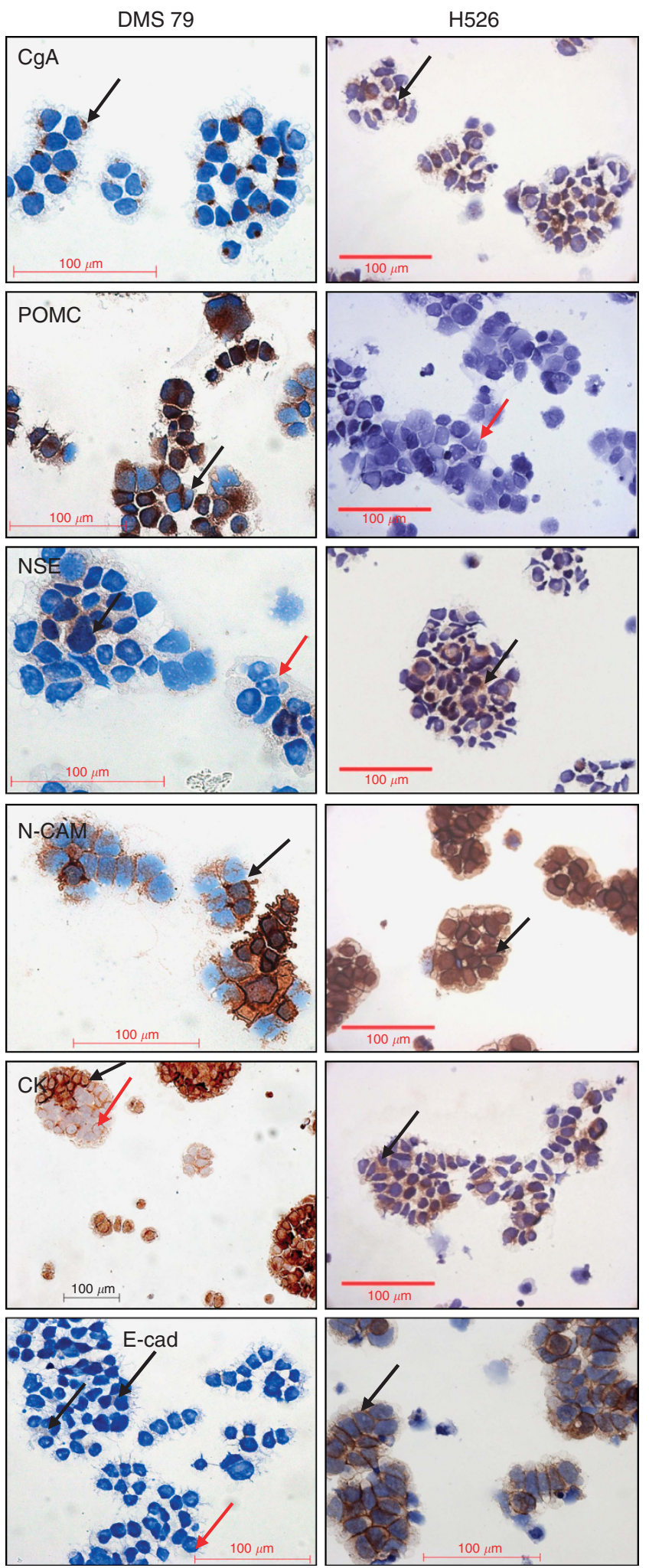

Figure 4. Neuroendocrine and epithelial markers in the SCLC DMS 79 cell line (left panel) and $\mathrm{NCl}-\mathrm{H} 526$ cell line (right panel). Cells were prepared as cytospins and stained for neuroendocrine markers, chromogranin A ( $\mathrm{CgA})$, pro-opiomelanocortin (POMC), neuron-specific enolase (NSE), CD56 (NCAM) neural cell adhesion molecule and epithelial markers cytokeratin (CK) and e-cadherin (E-Cad). Black arrows indicate examples of brown, positive staining and red arrows no staining. All results are representative of three experiments.
POMC and CK across xenografts and marked staining for both markers in tumour cells infiltrating into the surrounding capsule and muscle tissue. The dual neuroendocrine and epithelial phenotype has previously been described in patient samples, but our data suggest that the dual phenotype of SCLC cells is also retained in local invasion and possibly metastasis. The neuroendocrine origin of SCLC is widely recognised, but how this relates to the epithelial characteristics of this tumour is not clear. Until this interaction is understood, it will be difficult to determine how the phenotype affects metastasis and the tumour's response to the treatment regimens of chemotherapy and irradiation.

There is evidence that patients with features of the ectopic ACTH syndrome have high levels of POMC in the circulation (White and Clark, 1993) and some of these patients have SCLC (Stewart et al, 1994). This paper reports the first validated robust GCP method for quantifying POMC in patients without clinical symptoms of ectopic ACTH syndrome. This led to the finding that a subset of patients with SCLC, but without obvious evidence of ectopic ACTH syndrome, had concentrations of POMC, which were above the range found in normal subjects (Gibson et al, 1994; Russell et al, 2010). Given the evidence that SCLC tumours have neuroendocrine features, it is perhaps surprising that only a low percentage of these patients secrete POMC; however, tumours may still possess other neuroendocrine characteristics without producing POMC. This is supported by studies showing that another prohormone, proGRP, is present in approximately $70 \%$ of patients with SCLC (Molina et al, 2004a,b).

There was significantly worse overall survival in the patients with elevated circulating levels of POMC. In this group of patients, we also found a correlation between CTC number and circulating POMC. Circulating tumour cells are known to be indicative of poor prognosis, suggesting that POMC levels may also have a prognostic role. However, this study was conducted on a relatively small patient cohort. Further studies are warranted involving a larger number of patients to determine whether the association between POMC, CTCs and liver metastasis is important in SCLC progression. In addition, if we can elucidate how POMC is elevated in this subset of patients and how this relates to tumour progression, we may develop a greater understanding of this disease, ultimately allowing us to develop more successful diagnostics and therapeutics.

Higher levels of circulating POMC were found in our patients with liver metastasis compared to patients with metastases at other sites. This finding complements work on a mouse model of SCLC, showing distinct neuroendocrine and non-neuroendocrine heterogeneity. The authors found that a mixed population of neuroendocrine and non-neuroendocrine cells injected into mice to form xenografts were essential for metastases to develop in the liver. Furthermore, liver metastases consisted solely of neuroendocrinedifferentiated cells, with no evidence of non-neuroendocrine cells despite both cell types being delivered concomitantly. No metastases were detected when either neuroendocrine or nonneuroendocrine cells were injected alone (Meuwissen et al, 2003; Calbo et al, 2011). There are neuroendocrine biomarkers that are more sensitive indicators of SCLC, such as pro-GRP. However, the analysis of novel biomarkers is essential if we are to improve the understanding of the disease. The discovery of a biomarker able to predict metastasis to a particular organ could prove extremely beneficial in improving treatment regimens and overall survival.

Although the presence of heterogeneity within other tumour types is widely accepted, this often refers to epithelial and mesenchymal phenotypes. Where and how neuroendocrine characteristics fit in the characterisation of heterogeneity in SCLC has yet to be fully elucidated. However, given that in the mouse model of SCLC, neuroendocrine features are important in tumour progression and metastasis (Calbo et al, 2011), there may be cooperativity between epithelial and neuroendocrine cells, with the 

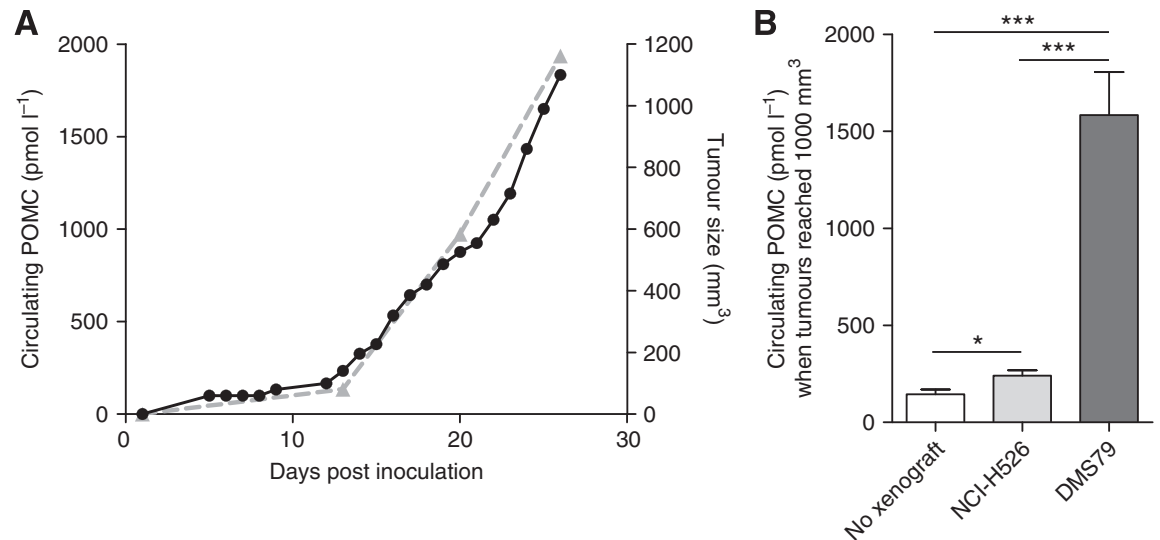

Figure 5. POMC secretion and tumour growth in a xenograft model. (A) DMS 79 cells were injected s.c. into nude mice at $5 \times 10^{6}$ per mouse in matrigel. Tumour growth was measured daily (black line). Plasma was collected via tail nick sampling every 7 days from day 13 in order to measure circulating POMC (grey dashed line). Results are representative of five mice. (B) NCl-H526 xenografts were established using the same protocol. Plasma was collected from nude mice with no tumour (no xenograft), $n=9$, and NCl-H526, $n=8$, and DMS 79, $n=5$ xenografts. Tumours were excised when they reached $1000 \mathrm{~mm}^{3}$. ${ }^{\star} P<0.05 ;{ }^{\star \star \star} P<0.001$.
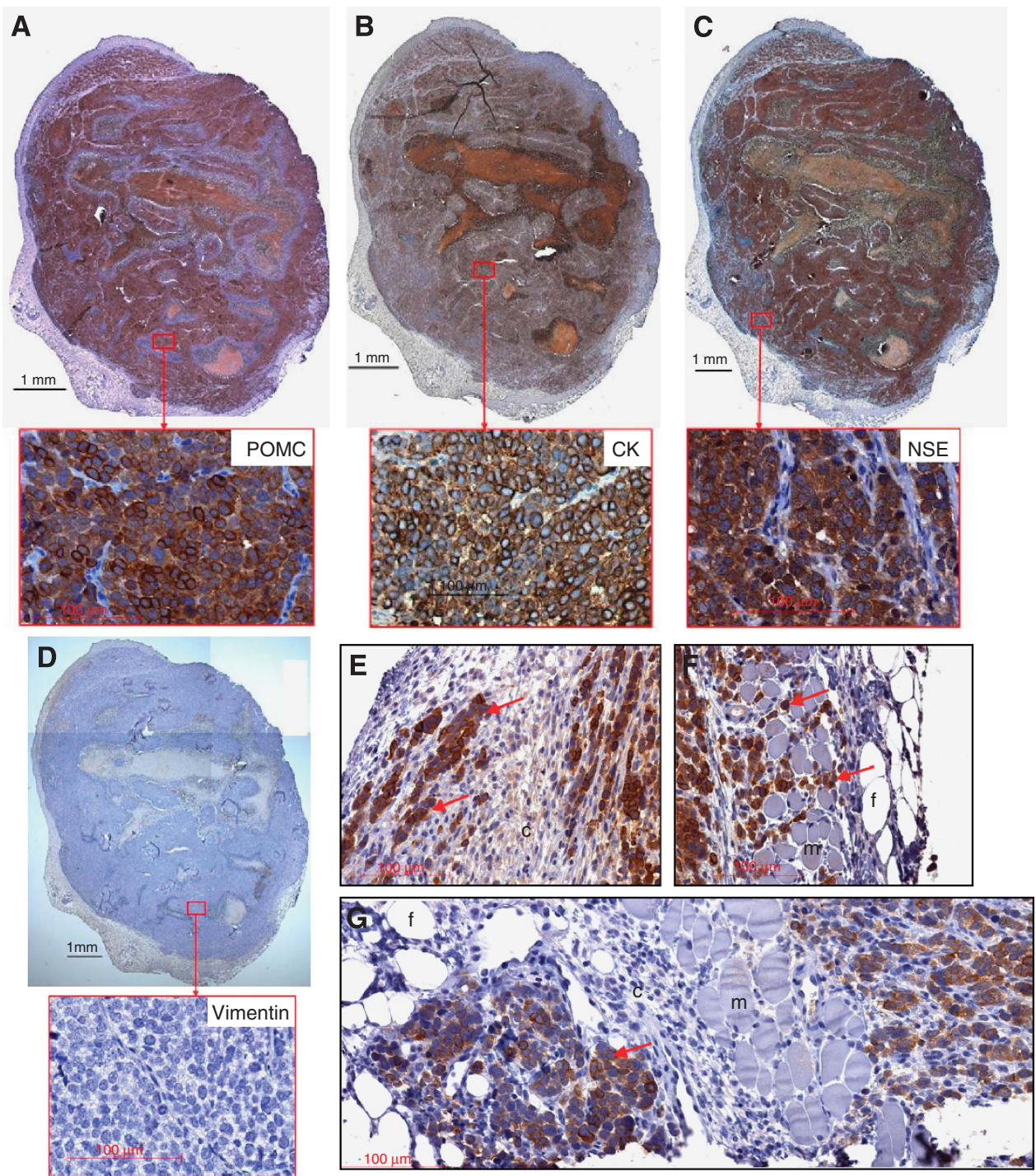

Figure 6. DMS 79 xenograft tumours retain their neuroendocrine phenotype in vivo and show local invasion into the capsule. DMS 79 tumours were excised at $1000 \mathrm{~mm}^{3}$ and whole sections stained for POMC (A), cytokeratin (B), NSE (C) and vimentin (D). POMC DAB staining revealed tumour cells that had invaded into the surrounding capsule/muscle tissue (E-G). Red arrows indicate tumour cells that have invaded into the capsule through muscle layers, or are actively invading through muscle. $m=$ myocytes, $c=$ fibrotic capsule, $f=$ fat deposits. 
latter as the critical cell type for metastasis formation (Calbo et al, 2011). In the panel of human SCLC cell lines we studied, more of the cell lines had evidence of epithelial markers than neuroendocrine characteristics. To pursue this further, we cloned the DMS 79 cell line and found that evidence of neuroendocrine/epithelial heterogeneity remained.

We have investigated the expression of POMC as a marker of a neuroendocrine phenotype, but in addition the clonal DMS 79 cells have other neuroendocrine features including dense core secretory granules and chromogranin A, NSE and CD56 (NCAM) expression. Furthermore, the SCLC cell line, H526, which is negative for POMC, stained positively for other neuroendocrine markers and for the epithelial markers, CK and E-cadherin, which confirms the presence of heterogeneity in markers between SCLC cell lines. The absence of POMC and the presence of other neuroendocrine markers in $\mathrm{H} 526$ cells are indications that SCLC cells can be neuroendocrine but not POMC positive. Therefore, it is important to consider a range of biomarkers when investigating the neuroendocrine phenotype in these cells.

The neuroendocrine phenotype was also present when DMS 79 cells were grown in vivo as a xenograft, as evidenced by the elevated levels of circulating POMC when compared with a non-POMC-secreting tumour control. This indicates that the increased POMC is coming from the tumour itself and not from pituitary-derived POMC, which would be highly unlikely as upregulation of the stress axis would increase secretion of ACTH rather than POMC. In addition, the tumours stained positively for $\mathrm{CK}$ and displayed a more uniform staining pattern than the cell line in vitro, indicating that the dual phenotype had prevailed in this model. Tumour cells with the dual phenotype were also found invading into the tumour capsule and through layers of muscle on the outer edge of the tumour, suggesting that neuroendocrine and epithelial characteristics are both required for local invasion in human SCLC cells. In contrast to the murine model developed in Anton Berns' group, we did not see in vivo heterogeneity with human SCLC cells in our model. Therefore, we suggest that rather than there being co-operation between cells of different phenotypes, it is important for human SCLC cells to possess a dual phenotype to promote invasion and metastasis. Further studies investigating the presence and distribution of metastases in vivo using murine lung cancer xenograft models are needed.

Overall this study has gathered evidence from several different approaches, which all suggest that neuroendocrine and epithelial features can coexist. The indirect evidence from patient samples of a correlation between circulating POMC and circulating epithelialpositive tumour cells is supported by the presence of both neuroendocrine and epithelial features in human SCLC tumour cells in vitro. Perhaps more importantly, this persists in the xenograft model, with the tumours displaying a dual neuroendocrine and epithelial phenotype, even in invading cells. Furthermore, in patients, measuring circulating POMC suggests that the neuroendocrine characteristics represent a more aggressive phenotype, particularly in terms of reduced survival and increased liver metastases.

\section{REFERENCES}

Abidin AZ, Garassino MC, Califano R, Harle A, Blackhall F (2010) Targeted therapies in small cell lung cancer: a review. Therap Adv Med Oncol 2: 25-37.

Baillie-Johnson H, Twentyman PR, Fox NE, Walls GA, Workman P, Watson JV, Johnson N, Reeve JG, Bleehen NM (1985) Establishment and characterisation of cell lines from patients with lung cancer (predominantly small cell carcinoma). Br J Cancer 52: 495-504.
Calbo J, van Montfort E, Proost N, van Drunen E, Beverloo HB, Meuwissen R, Berns A (2011) A functional role for tumor cell heterogeneity in a mouse model of small cell lung cancer. Cancer Cell 19: 244-256.

Clark L, White A (1994) Ectopic hormonal disorders. Curr Opin Endocrinol Diabet 1: 56-59.

Crosby SR, Stewart MF, Ratcliffe JG, White A (1988) Direct measurement of the precursors of adrenocorticotropin in human plasma by two-site immunoradiometric assay. J Clin Endocrinol Metab 67: 1272-1277.

Gibson S, Crosby SR, Stewart MF, Jennings AM, McCall E, White A (1994) Differential release of proopiomelanocortin-derived peptides from the human pituitary: evidence from a panel of two-site immunoradiometric assays. J Clin Endocrinol Metab 78: 835-841.

Hou JM, Krebs M, Ward T, Sloane R, Priest L, Hughes A, Clack G, Ranson M, Blackhall F, Dive C (2011) Circulating tumor cells as a window on metastasis biology in lung cancer. Am J Pathol 178: 989-996.

Jemal A, Siegel R, Ward E, Hao Y, Xu J, Thun MJ (2009) Cancer statistics, 2009. CA Cancer J Clin 59: 225-249.

Meuwissen R, Linn SC, Linnoila RI, Zevenhoven J, Mooi WJ, Berns A (2003) Induction of small cell lung cancer by somatic inactivation of both Trp53 and Rb1 in a conditional mouse model. Cancer Cell 4: 181-189.

Molina R, Auge JM, Alicarte J, Filella X, Vinolas N, Ballesta AM (2004a) Pro-gastrin-releasing peptide in patients with benign and malignant diseases. Tumour Biol 25: 56-61.

Molina R, Filella X, Auge JM (2004b) ProGRP: a new biomarker for small cell lung cancer. Clin Biochem 37: 505-511.

Oliver RL, Davis JR, White A (2003) Characterisation of ACTH related peptides in ectopic Cushing's syndrome. Pituitary 6: 119-126.

Ratcliffe JG, Podmore J, Stack BH, Spilg WG, Gropp C (1982) Circulating ACTH and related peptides in lung cancer. $\mathrm{Br} J$ Cancer 45: 230-236.

Riaz SP, Luchtenborg M, Coupland VH, Spicer J, Peake MD, Moller H (2012) Trends in incidence of small cell lung cancer and all lung cancer. Lung Cancer 75: 280-284.

Russell GM, Henley DE, Leendertz J, Douthwaite JA, Wood SA, Stevens A, Woltersdorf WW, Peeters BW, Ruigt GS, White A, Veldhuis JD, Lightman SL (2010) Rapid glucocorticoid receptor-mediated inhibition of hypothalamic-pituitary-adrenal ultradian activity in healthy males. J Neurosci 30: 6106-6115.

Stewart PM, Gibson S, Crosby SR, Pennt R, Holder R, Ferry D, Thatcher N, Phillips P, London DR, White A (1994) ACTH precursors characterize the ectopic ACTH syndrome. Clin Endocrinol 40: 199-204.

Stovold R, Blackhall F, Meredith S, Hou J, Dive C, White A (2012) Biomarkers for small cell lung cancer: neuroendocrine, epithelial and circulating tumour cells. Lung Cancer 76: 263-268.

Tibbe AG, de Grooth BG, Greve J, Liberti PA, Dolan GJ, Terstappen LW (1999) Optical tracking and detection of immunomagnetically selected and aligned cells. Nat Biotechnol 17: 1210-1213.

Travis W, Brambilla E, Muller-Hermelink H, Harris C (2004) Pathology and Genetics of Tumours of the Lung, Pleura, Thymus and Heart (IARC WHO Classification of Tumours). IARC Press, Oxford University Press.

Travis WD (1999) Neuroendocrine tumours of the lung. In Lung Tumours: Fundamental Biology and Clinical Management, Brambilla C, Brambilla E eds. Vol. 124, pp 29-61. Marcel Dekker: New York, NY, USA.

Trump DL, Abeloff MD, Hsu TH (1982) Frequency of abnormalities of cortisol secretion and water metabolism in patients with small cell carcinoma of the lung and other malignancies. Chest 81: 576-580.

White A, Clark AJ (1993) The cellular and molecular basis of the ectopic ACTH syndrome. Clin Endocrinol 39: 131-141.

White A, Stewart MF, Farrell WE, Crosby SR, Lavender PM, Twentyman PR, Rees LH, Clark AJ (1989) Pro-opiomelanocortin gene expression and peptide secretion in human small-cell lung cancer cell lines. $J \mathrm{Mol}$ Endocrinol 3: 65-70.

This work is published under the standard license to publish agreement. After 12 months the work will become freely available and the license terms will switch to a Creative Commons AttributionNonCommercial-Share Alike 3.0 Unported License. 